\title{
Cannabis-Induced Catatonia: A Case Series
}

\author{
Hema Mekala ${ }^{1}$, Zamaar Malik ${ }^{2}$, Judith Lone ${ }^{2}$, Kaushal Shah ${ }^{1}$, Muhammad Ishaq ${ }^{1}$ \\ 1. Psychiatry, Griffin Memorial Hospital, Norman, USA 2. Psychiatry, Medical University of the Americas, Camps, KNA
}

Corresponding author: Kaushal Shah, kpshahmd@gmail.com

\begin{abstract}
Catatonia is a psychomotor condition characterized by physical presentations ranging from severe immobility to excessive psychomotor agitation with an array of accompanying emotional aspects. Though initially thought to be a subform of schizophrenia, it is now recognized to be associated with many different psychiatric, neurological, and medical diagnoses. The emergence of catatonia is becoming more prevalent with its changing pattern and extensive use of recreational and illegal drugs. With the legalization of marijuana, its use is on the rise leading to several mental health conditions, including catatonia. If left untreated, catatonia has a significant morbidity and mortality rate; hence, prompt evaluation and diagnosis are critical for the prevention of adverse events. Benzodiazepines (BZDs) and electroconvulsive therapy (ECT) have been found to be most effective and remained as the preferred treatment options. In this paper, we present the case of two patients who presented with catatonia after cannabis consumption and discuss
\end{abstract} their treatment course and management.

Categories: Emergency Medicine, Neurology, Psychiatry

Keywords: synthetic cannabis, k2, cannabis, catatonia, addiction, thc, schizophrenia, benzodiazepine, ect, psychiatry

\section{Introduction}

Catatonia is a common condition observed in acutely ill patients with mental health conditions. In the United States, approximately 90,000 people tend to suffer from catatonia each year, with a prevalence ranging between $7.6 \%$ to $38 \%$ [1]. Catatonia is mostly evidenced in patients with delirium, mood disorders, schizophrenia, post-traumatic stress disorder (PTSD), and dissociative states [1,2]. It is most often characterized by decreased motor activity, lack of responsiveness, and posturing. Furthermore, it is classified into three categories, namely retarded, malignant, and excited catatonia [3]. Catatonia may also occur due to metabolic, neoplastic, infectious conditions, withdrawal or intoxication of prescription medications, and recreational drug use [2,3]. It is considered a medical and psychiatric emergency leading to severe rigidity, autonomic nervous system instability, and altered mental status resulting in death if untreated $[1,2]$. Even though diagnosing catatonia is straightforward from the clinical presentation, the etiology is often overlooked. This case series places emphasis mainly on cannabis-induced catatonia when considering a differential diagnosis.

Received 05/28/2020

Review began 06/05/2020 Review ended 06/06/2020 Published 06/13/2020

() Copyright 2020 Mekala et al. This is an open access article distributed under the terms of the Creative Commons Attribution License CC-BY 4.0., which permits unrestricted use, distribution, and reproduction in any medium, provided the original author and source are credited.

\section{Case Presentation}

\section{Case 1}

A 22-year-old Caucasian woman with no known past psychiatric history was transferred from another facility for depression, suicidal ideation, psychosis, and bizarre behavior. On admission, she was withdrawn with minimal verbalization, despondent, had a flat affect, and exhibited thought blocking throughout the evaluation. Physical examination and laboratory studies showed no additional findings except for anemia and positive urine drug screen for marijuana.

After a thorough clinical evaluation, catatonia was considered as the underlying cause for her presentation, and she was started on lorazepam $0.5 \mathrm{mg}$ by mouth (PO) every morning (QAM), sertraline $50 \mathrm{mg}$ PO QAM (by mouth every morning), and olanzapine $5 \mathrm{mg}$ by mouth (PO) each night at bedtime (QHS). She was later discontinued on sertraline and instead started on duloxetine $30 \mathrm{mg}$ PO QAM (by mouth every morning), which was gradually titrated up to $120 \mathrm{mg}$ PO QAM (by mouth every morning). The lorazepam was also increased to $1 \mathrm{mg}$ by mouth (PO) twice a day (BID). The new regimen resulted in a significant improvement in her mood symptoms and resolution of catatonia. After recovery, the patient reported that she had no previous history of psychiatric and medical conditions and was not on any medications prior to her admission. She admitted to smoking marijuana daily to cope with the "stress" in her life for the past few years. Hence, in the absence of any other etiology, it was concluded that the patient's catatonic presentation, depression, and psychosis were attributed to her cannabis use.

\section{Case 2}

A 33-year-old Caucasian woman with a past psychiatric history of schizophrenia and depression was brought to the hospital by her husband for the inability to take care of herself. He reported that the patient was having bizarre disorganized behavior and a worsening of her baseline auditory hallucinations. 
According to her family, the patient was admitted to another facility for schizophrenia and was discharged ten days ago. Her medication compliance was unknown. On admission, the patient was unable to sit or talk, mumbled sometimes, and had a flat affect. We continued her home medication, olanzapine 5 mg PO BID (by mouth twice a day), and started her on lorazepam $1 \mathrm{mg}$ PO BID (by mouth twice a day) for possible catatonia. The patient improved gradually and was able to communicate as her catatonia resolved. Upon further evaluation, the patient reported that after discharge from another facility, she smoked marijuana resin daily and did not know the events that led to her admission. Her worsening of psychosis and the onset of catatonia were associated with resin use.

\section{Discussion}

Though the presentation of catatonia is evident in the case scenarios discussed above, identifying possible etiologies and preventing its recurrence was clinically challenging as it could occur due to a wide variety of medical and psychiatric conditions.

With increasing legalization and marijuana use worldwide, including in the United States, we are likely to see an increased prevalence of catatonia along with other psychiatric conditions [4,5]. Cannabis products can be consumed in a number of ways, and they all have different potencies. The potency of cannabis depends on the concentration (\%) of $\Delta 9$-tetrahydrocannabinol (THC), which is the primary psychoactive component of the cannabis plant [6]. Different products of cannabis such as "buds" (dried cannabis flowers), oil (butane honey oil, shatter, wax, crumble), and resin (hashish, bubble hash) are available, and they are consumed either through smoking or as edibles [7].

Although no precise pathophysiological mechanisms of catatonia have been identified, studies have reported that it occurs primarily due to decreased activity of gamma-aminobutyric acid type $A\left(G_{B A A}\right)$ receptor, dopamine at the dopamine type-2 (D2) receptor, and glutamate at N-methyl-D-aspartate (NMDA) receptor [8]. A study on mice conducted by Fairbairn et al. showed that oral THC resulted in cataleptic events due to the increased availability of arachidonic acid, possibly from the gut, with a subsequent increase in the level of prostaglandin E2 (PGE2) [9]. Consumption of synthetic cannabinoid, also known as "spice" or "K2," has also led to catatonic presentations. Synthetic cannabinoids have had increased popularity in recent years due to their ease of availability, low cost, and lack of detection by standard urine drug screens [10]. A case report by Smith et al. mentioned that a 17-year-old male with no previous psychiatric history presented with catatonic symptoms after smoking about $2 \mathrm{~g}$ to $3 \mathrm{~g}$ per day of $\mathrm{K} 2$, which was resolved with lorazepam administration and six electroconvulsive therapy (ECT) treatments [11]. However, both of our patients denied using synthetic cannabinoids.

Benzodiazepines (BZDs), especially lorazepam, are found to be extremely beneficial in resolving symptoms of different catatonic presentations by increasing GABA and decreasing NMDA receptor activity and are generally used as the first line of treatment [12]. Even though it is rare, the dosing of BZDs up to $20 \mathrm{mg}$ to 30 $\mathrm{mg}$ per day is required for improvement or resolution of catatonic symptoms [13]. In patients unresponsive to BZDs, ECT has shown to be efficacious. Along with these interventions, avoiding exacerbating factors such as recreational or illegal drug use and dopamine blocking agents may help relieve catatonia symptoms [12].

\section{Conclusions}

Although there is limited evidence thus far from the literature regarding cannabis use as a potential cause of catatonia, physicians should consider it as a potential etiology, as it is now one of the most commonly abused substances. Catatonia presentation could be a traumatic experience for the patients and their families. Hence, it is crucial to educate them about the potential risks associated with marijuana consumption and counsel against its use. Above all, clinicians should be vigilant about the use of any recreational drugs by their patients to provide optimal care.

\section{Additional Information \\ Disclosures}

Human subjects: Consent was obtained by all participants in this study. Conflicts of interest: In compliance with the ICMJE uniform disclosure form, all authors declare the following: Payment/services info: All authors have declared that no financial support was received from any organization for the submitted work. Financial relationships: All authors have declared that they have no financial relationships at present or within the previous three years with any organizations that might have an interest in the submitted work. Other relationships: All authors have declared that there are no other relationships or activities that could appear to have influenced the submitted work.

\section{References}

1. Bhati MT, Datto CJ, O’Reardon JP: Clinical manifestations, diagnosis, and empirical treatments for catatonia. Psychiatry. 2007, 4:46-52.

2. Prajapati PR, Fabius D, Pradhan B: Clinical course and management of drug-induced catatonia and paranoid 


\section{Cureus}

behavior in an adolescent. Klinik Psikofarmakol Bülteni. 2015, 26:310-315. 10.5455/bcp.20151217105437

3. Fink M, Taylor MA: The catatonia syndrome: forgotten but not gone. Arch Gen Psychiatry. 2009, 66:11731177. 10.1001/archgenpsychiatry.2009.141

4. Radhakrishnan R, Wilkinson ST, D'Souza DC: Gone to pot - a review of the association between cannabis and psychosis. Front Psychiatry. 2014, 5:10.3389/fpsyt.2014.00054

5. Hasin DS, Saha TD, Kerridge BT, et al.: Prevalence of marijuana use disorders in the United States between 2001-2002 and 2012-2013. JAMA Psychiatry. 2015, 72:1235-1242. 10.1001/jamapsychiatry.2015.1858

6. ElSohly MA, Mehmedic Z, Foster S, Gon C, Chandra S, Church JC: Changes in cannabis potency over the last 2 decades (1995-2014) - analysis of current data in the United States. Biol Psychiatry. 2016, 79:613-619. 10.1016/j.biopsych.2016.01.004

7. National Academies of Sciences, Engineering, and Medicine: The Health Effects of Cannabis and Cannabinoids: The Current State of Evidence and Recommendations for Research. The National Academies Press, Washington DC; 2017. 10.17226/24625

8. Carroll BT: The universal field hypothesis of catatonia and neuroleptic malignant syndrome . CNS Spectr. 2000, 5:26-33. 10.1017/s1092852900013365

9. Fairbairn JW, Pickens JT: The oral activity of delta'-tetrahydrocannabinol and its dependence on prostaglandin e2. Br J Pharmacol. 1979, 67:379-385. 10.1111/j.1476-5381.1979.tb08691.x

10. Martz G, Tankersley W, Mekala HM, Motiwala H: Rates of synthetic cannabinoid use in adolescents admitted to a treatment facility. Prim Care Companion CNS Disord. 2018, 20:17m02265. 10.4088/PCC.17m02265

11. Smith DL, Roberts C: Synthetic marijuana use and development of catatonia in a 17-year-old male . Minn Med. 2014, 97:38.

12. Sienaert P, Dhossche DM, Vancampfort D, De Hert M and Gazdag G: A clinical review of the treatment of catatonia. Front Psychiatry. 2014, 5:181. 10.3389/fpsyt.2014.00181

13. Luchini F, Medda P, Mariani MG, Mauri M, Toni C, Perugi G: Electroconvulsive therapy in catatonic patients: efficacy and predictors of response. World J Psychiatr. 2015, 5:182-192. 10.5498/wjp.v5.i2.182 